\title{
Ultra-low-noise ultrafast fiber lasers
}

\author{
Alexander M. Heidt \\ Institute of Applied Physics, University of Bern, Sidlerstrasse 5, 3012 Bern, Switzerland
}

Femtosecond lasers based on mature Erbium-doped fiber (Er:fiber) technology have become the workhorse for ultrafast photonics research and applications due their stability, compactness, and ease of use. Commercial ultrafast Er:fiber lasers are available that have been developed specifically to exhibit ultra-low amplitude and phase noise, exhibiting relative intensity noise (RIN) values far below $0.1 \%$, making them essential tools for demanding applications such as ultrafast spectroscopy or precision metrology [1].

A convenient approach of extending the excellent performance of Er:fiber lasers to other wavebands, e.g. into the $2 \mu \mathrm{m}$ regime, is their nonlinear spectral broadening in nonlinear fibers, followed by amplification in a Thuliumor Holmium-doped fiber amplifier system. However, previous implementations using conventional nonlinear fibers pumped in the anomalous dispersion (AD) regime suffered from the generation of excess noise via quantum, technical, and polarization noise amplification by the involved nonlinear processes, resulting in RIN values in the range $0.3-0.7 \%$, i.e. about an order of magnitude higher than the corresponding Er:fiber system This excess noise has recently been identified as the major performance limiting factor for the further development of high-power frequency comb sources at $2 \mu \mathrm{m}[2]$.

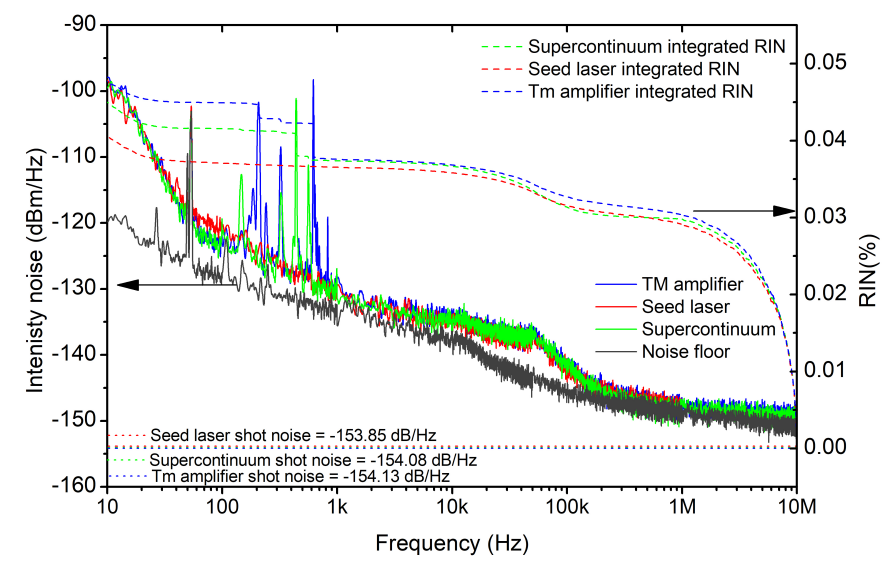

Fig. 1: Measured noise characteristics of the Er:fiber seed laser (red), ANDi supercontinuum (green), and Tm:fiber amplifier (blue) [5].

In this contribution I discuss the conditions required for suppressing this excess noise generation during nonlinear spectral broadening, present specialty optical fibers specifically designed for this purpose, and review the latest developments in their application aimed at realizing the next generation of ultra-low noise frequency combs and ultrashort pulse sources in the $2 \mu \mathrm{m}$ spectral region. It is shown that ultra-low noise nonlinear spectral broadening and supercontinuum (SC) generation is enabled by all-normal dispersion (ANDi) fibers, which effectively suppress quantum and polarization noise amplification and lead to nonlinear dynamics that are extremely robust against technical noise [3,4]. The application of these ANDi SC sources for the coherent seeding of ultrafast Thulium- / Holmium-doped fiber amplifiers is demonstrated to result in an order-of-magnitude reduction of RIN to $<0.05 \%$, i.e. virtually identical to the driving Er:fiber laser (Fig. 1) [5]. Therefore, neither the nonlinear spectral broadening nor the amplification process introduces significant excess noise, highlighting the potential of ANDi SC seed sources for the next generation of ultra-low noise, broadband, high-power ultrafast fiber amplifiers and frequency combs.

\section{References}

[1] D. Brida, G. Krauss, A. Sell, and A. Leitenstorfer, "Ultrabroadband Er:fiber lasers," Laser Photonics Rev. 8, 409 (2014).

[2] C. Gaida et al., "High-power frequency comb at $2 \mu \mathrm{m}$ wavelength emitted by a Tm-doped fiber laser system," Opt. Lett. 43, 5178 (2018).

[3] A. M. Heidt, J. S. Feehan, J. H. V. Price, and T. Feurer, "Limits of coherent supercontinuum generation in normal dispersion fibers," J. Opt. Soc. Am. B 34, 764-775 (2017).

[4] B. Sierro and A. M. Heidt, "Noise amplification in all-normal dispersion fiber supercontinuum generation and its impact in ultrafast photonics applications," OSA Continuum (in press).

[5] A. Rampur, Y. Stepanenko, G. Stepniewski, T. Kardaś, D. Dobrakowski, D.-M. Spangenberg, T. Feurer, A. Heidt, and M. Klimczak, "Ultra low-noise coherent supercontinuum amplification and compression below $100 \mathrm{fs}$ in an all-fiber polarization-maintaining thulium fiber amplifier,” Opt. Express 27, 35041-35051 (2019). 